\title{
On the Throughput of Wireless Interference Networks with Limited Feedback
}

\author{
Hamed Farhadi, Chao Wang, Mikael Skoglund \\ Communication Theory Laboratory, School of Electrical Engineering \\ Royal Institute of Technology(KTH), Stockholm, Sweden
}

\begin{abstract}
Considering a single-antenna $M$-user interference channel with symmetrically distributed channel gains, when the channel state information (CSI) is globally available, applying the ergodic interference alignment scheme, each transmitterreceiver pair achieves a rate proportional to $1 / 2$ of a single user's interference-free achievable rate. This is substantially higher than the achievable rate of the conventional orthogonal transmission schemes such as TDMA. Since the rigid requirement on the CSI may be difficult to realize in practice, in this paper we investigate the performance of applying the ergodic interference alignment scheme when the estimation of each channel gain is made globally known through exploiting only a limited feedback signal from the associated receiver of that channel. Under a block fading environment, we provide a lower bound on the achievable average throughput of the network. Our results imply that the better performance of interference alignment over TDMA may still exist even without the assumption of perfect CSI. Also, the trade off between allocating feedback rate of each receiver to the desired channel or the interference channels at deferent SNR region investigated.
\end{abstract}

\section{INTRODUCTION}

The performance limits of the interference channels (ICs) have attracted much interest for decades, e.g. the capacity region of the two-user IC has been the subject of extensive research. Although certain achievable rates and outer bounds on the capacity region of the two-user IC have been proposed, the exact capacity region is still unknown in general [1], [2]. Extension of the results on the two-user IC to general $M$-user ICs is even more complicated. Recently, a novel technique called interference alignment [3], [4] showed that such networks may not be interference limited in high signal-to-noise ratio (SNR) region. It has been shown that through properly aligning the interference at each receiver, the achievable sum rate of an $M$-user IC can be $\frac{M}{2} \log (\mathrm{SNR})+o(\log (\mathrm{SNR}))$ for time-varying (or frequency-selective) channels [3]. This achievable sum rate linearly scales with the number of users at high SNR and is substantially higher than that of the time division multiple access (TDMA) scheme, which is only $\log (\mathrm{SNR})+o(\log (\mathrm{SNR}))$. Furthermore, when the channel gains are symmetrically distributed, ergodic interference alignment scheme has been developed in [5] so that the sum rate of $\frac{M}{2} E\left[\log \left(1+2|h|^{2} \mathrm{SNR}\right)\right]$ is achievable under an ergodic setting. Such a result implies that the IC under time-varying channel may not be interference limited at any SNR.

To achieve the outstanding performance promised by the aforementioned schemes, in general the channel state information (CSI) is assumed to be perfectly known at all the receivers and transmitters. Since acquiring such perfect CSI is a chal- lenging problem, references [5], [6], [7] have investigated the case when each receiver provides only the quantized version of its incoming channel gains to the other terminals through feedback signals. It has been shown that when the quantization resolutions are sufficiently high, the good performance is still achievable [5], [6]. However, the bandwidth of the feedback channels may be limited in practice such that the terminals may not be able to attain a sufficiently accurate estimations of the CSI. Therefore, in this paper we study the performance of applying the ergodic interference scheme when the quantizers deployed at each receiver have only limited resolutions. Under a time-varying block-fading channel we provide a lower bound on the achievable throughput of the system.

Our results show that even with a limited number of feedback bits the throughput of the network applying interference alignment can still be larger than that achieved by TDMA. It can also be observed that in the low SNR region equipping a higher-resolution quantizer for the desired channel at each receiver is more preferable. This is because the network is basically noise limited at low SNR and conducting efficient rate allocation according to the desired channel gain of each transmitter has more impact on the network throughput. On the other hand, in the high SNR region since the interference dominantly affects the achievable throughput, it is better to provide each receiver higher-resolution quantizers for the interference channels to more accurately align the interference.

\section{SYSTEM MODEL}

We consider a single-antenna $M$-user interference network represented in Fig. 1. Each transmitter has independent messages for its dedicated receiver. Since all transmitters share the transmission medium, each of the receivers gets the desired message from the corresponding transmitter over the desired channel and also receives interference from all other transmitters over interference channels. We assume that the users communicate over discrete-time, block-fading (each block contains $n$ channel uses) channels. The channel gains remain fixed over each block, but change independently across different blocks. We consider the transmission over a large number of blocks.

At any block index $a$, the transmitter $k$ chooses its message independently and uniformly from a message set of size $2^{n R_{k}^{a}}$ where $R_{k}^{a} \geq 0$ is the code rate. It encodes its message to codeword $x_{k}^{a}$ with length $n$. We assume that the codeword length $n$ is sufficiently large and the code is capacity achieving. 


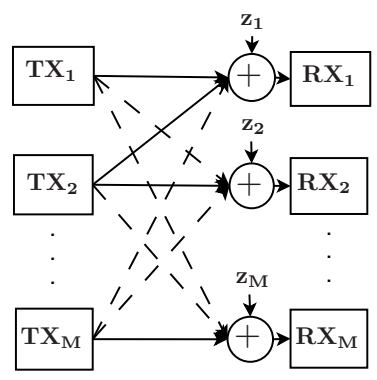

Fig. 1: System model

The channel output at the receiver $k$ is given by:

$$
y_{k}^{a}=h_{k k}^{a} x_{k}^{a}+\sum_{l=1, l \neq k}^{M} h_{k l}^{a} x_{l}^{a}+z_{k}^{a} \quad, \quad k=1,2, \ldots, M
$$

where $z_{k}^{a}$ is the unit-power additive white Gaussian noise (AWGN) and $h_{k l}^{a}$ is the channel gain between transmitter $l$ and receiver $k$ drawn independently from a Rayleigh distribution, i.e. $h_{k l}^{a} \sim \mathcal{C N}(0,1)$. We assume channels have limited gain $\left|\Re\left[h_{k l}^{a}\right]\right|<h_{\max }$ and $\left|\Im\left[h_{k l}^{a}\right]\right|<h_{\max }$ in which $h_{\max }$ is a constant. Each transmitter satisfies an average power constraint $P_{k}=E\left[\left|x_{k}^{a}\right|^{2}\right] \leq P_{k}^{\max }$, in which $P_{k}^{\max }$ is the maximum transmission power of the transmitter $k$. The first term in the right hand side (RHS) of (1) is the desired signal for receiver $k$ and the second term is the interference.

\section{A. Transmission scheme}

We consider a transmission scheme similar to the ergodic interference alignment scheme proposed in [5]. At the beginning of each block, each receiver estimates the incoming channel gains based on training sequences (this estimation is assumed to be perfect). Next, it quantizes the channel gains and broadcasts the corresponding indices to all the other terminals. The quantized channel gain between transmitter $l$ and receiver $k$ at block $a$ is denoted by $\hat{h}_{k l}^{a}$ and the corresponding quantization error is denoted by $\delta_{k l}^{a}$. If at the block indices $m$ and $m_{p}$ the following conditions are satisfied:

$$
\hat{h}_{i i}^{m}=\hat{h}_{i i}^{m_{p}}, \hat{h}_{i j}^{m}=-\hat{h}_{i j}^{m_{p}} ; \forall i, j \in\{1, \ldots, M\}, i \neq j
$$

the channel pairs are called complement. It has been proved in [5] that for channels with a symmetric distribution, as long as number of channel realizations increases, for each block index $m$ the probability of finding block index $m_{p}$ such that the conditions (2) are satisfied increases. Therefore, for long enough block realizations almost surely we can find the pair of block indices such that the channels are complement.

Assume $m$ and $m_{p}$ are the block indices of a pair of complement channels. We require each transmitter to send the same codeword overs these two blocks $\left(x_{k}^{m}=x_{k}^{m_{p}}\right)$ Each receiver adds the received signals $\left(\bar{y}_{k}^{m}=y_{k}^{m}+y_{k}^{m_{p}}\right)$ and decode the codeword. Therefore, according to the system model in (1) the equivalent received signal $\bar{y}_{k}^{m}$ is:

$$
\bar{y}_{k}^{m}=\left(2 \hat{h}_{k k}^{m}+\delta_{k k}^{m}+\delta_{k k}^{m_{p}}\right) x_{k}^{m}+\sum_{l=1, l \neq k}^{M}\left(\delta_{k l}^{m}+\delta_{k l}^{m_{p}}\right) x_{l}^{m}+\bar{z}_{k}
$$

in which the first term in the RHS is the desired signal, the second term is the residual interference and $\bar{z}_{k}^{m}=z_{k}^{m}+z_{k}^{m_{p}}$. It has been proved in [5] that if the quantization resolution asymptotically goes to infinity, the power of the residual interference approaches zero and the ergodic interference alignment scheme achieves the rate tuple $\left(R_{1}, R_{2}, \ldots, R_{M}\right)$ for $R_{k}=\frac{1}{2} E\left[\log \left(1+2\left|h_{k k}\right|^{2} P_{k}\right)\right]$. This result shows that each transmitter-receiver pair achieves a rate proportional to $1 / 2$ of a single user's interference-free achievable rate. This is substantially higher than the achievable rate of the conventional orthogonal transmission schemes such as TDMA especially as the number of users $M$ increases.

For limited-resolution quantizers, as (3) shows quantization errors lead to a certain amount of the interference and some uncertainty on the desired channel gain. In the rest of the paper, we will study how the quantization error would affect the throughput of the network.

\section{B. Quantization and feedback scheme}

We consider uniform quantization [8]. There are two uniform quantizers associated to each channel, one for real part and one for imaginary part of the channel gain. Each quantizer maps the real (imaginary) part of the channel gain to the closest reconstruction point. For $K$ bit quantization, there are $2^{K}$ of such points uniformly spaced from $-h_{\max }$ to $h_{\max }$. Distance of the adjacent reconstruction points called quantizer step size and denoted by $\Delta=\frac{h_{\max }}{2^{K-1}}$. Usually, to quantize Gaussian random variables with variance $\sigma^{2}, h_{\max }=4 \sigma$ is an acceptable design value [9]. Therefore, we choose $h_{\max }=4$ for quantizing the unit-variance channel gains.

In general, the quantization of the desired and interference channels may have different resolutions. We assume that at each receiver there are two types of quantizers with different resolutions. Each of the two quantizers associated to the desired (interference) channel uses $K_{I}\left(K_{I I}\right)$ bits for quantization of the real or imaginary part of the channel gain. Therefore, the step size of the desired (interference) channel quantizers is $\Delta_{I}=\frac{h_{\max }}{2^{K I^{-1}}}\left(\Delta_{I I}=\frac{h_{\max }}{2^{K} I^{-1}}\right)$. Quantization errors bounded as $\frac{-\Delta_{I}}{2} \leq \Re\left[\delta_{k k}^{m}\right], \Re\left[\delta_{k k}^{m_{p}}\right], \Im\left[\delta_{k k}^{m}\right], \Im\left[\delta_{k k}^{m_{p}}\right] \leq \frac{\Delta_{I}}{2}$ and $\frac{-\Delta_{I I}}{2} \leq \Re\left[\delta_{k l}^{m}\right], \Re\left[\delta_{k l}^{m_{p}}\right], \Im\left[\delta_{k l}^{m}\right], \Im\left[\delta_{k l}^{m_{p}}\right] \leq \frac{\Delta_{I I}}{2} \forall k \neq l$, in which $\Re[x]$ and $\Im[x]$ denotes the real and imaginary parts of the random variable $x$, respectively.

At the beginning of each block, each receiver broadcasts $K_{f}=2 K_{I}+2(M-1) K_{I I}$ bits to all other terminals. Feedback channels assumed to be error free. Each terminal reconstructs the quantized channels based on the received feedback indices.

\section{OUTAGE ANALYSIS}

Assume that the channels with block indices $m$ and $m_{p}$ are complement. Requiring each transmitter to repeat the same codeword over the blocks $m$ and $m_{p}$, the signal to noise and interference ratio (SINR) of the equivalent received signal of user $k$ given in (3) is as follows:

$$
\operatorname{SINR}_{\bar{y}_{k}^{m}}=\frac{\left|2 \hat{h}_{k k}^{m}+\delta_{k k}^{m}+\delta_{k k}^{m_{p}}\right|^{2} P_{k}}{2+\sum_{l=1, l \neq k}^{M}\left|\delta_{k l}^{m}+\delta_{k l}^{m_{p}}\right|^{2} P_{l}} .
$$


The mutual information between the $k^{\text {th }}$ transmitter-receiver pair is $\frac{1}{2} \log \left(1+\operatorname{SINR}_{\bar{y}_{k}^{m}}\right)$. The pre-log factor $1 / 2$ appears due to the transmission of the same codeword over two blocks. Since the actual value of the $\operatorname{SINR}_{\bar{y}_{k}^{m}}$ is not known at the transmitter due to the random quantization errors, the exact value of the mutual information is unknown at the transmitter. If the chosen transmission rate $R_{k}^{m}$ is smaller than this mutual information, the decoding error probability can be made arbitrary small. Otherwise, the channel between transmitter-receiver $k$ is said to be in outage [10], and the outage probability is:

$$
\mathrm{P}_{k}^{\text {out }, m}=\operatorname{Pr}\left\{\frac{1}{2} \log \left(1+\operatorname{SINR}_{\bar{y}_{k}^{m}}\right)<R_{k}^{m}\right\} .
$$

It is difficult to find a closed-form expression of $\mathrm{P}_{k}^{\text {out }, m}$. Instead, we present an upper bound in the following theorem.

Theorem 1: If $R_{k}^{m}<R_{\max , k}^{m}$, the outage probability $\mathrm{P}_{k}^{\text {out }, m}$ defined in (5) can be upper bounded as:

$$
\mathrm{P}_{k}^{\text {out }, m} \leq \frac{1}{1+\left(r_{k}^{m}\right)^{2}}
$$

in which $R_{\max , k}^{m}=\frac{1}{2} \log \left(1+\frac{\left(12\left|\hat{h}_{k k}^{m}\right|^{2}+\Delta_{I}^{2}\right) P_{k}}{\Delta_{I I}^{2} \sum_{i=1, i \neq k}^{M} P_{i}+6}\right)$, and

$$
r_{k}^{m}=\frac{\frac{\left(12\left|\hat{h}_{k k}^{m}\right|^{2}+\Delta_{I}^{2}\right) P_{k}}{3\left(2^{\left.2 R_{k}^{m}-1\right)}-\frac{\Delta_{I I}^{2}}{3} \sum_{i=1, i \neq k}^{M} P_{i}-2\right.}}{\sqrt{\frac{7}{90} \Delta_{I I}^{4} \sum_{i=1, i \neq k}^{M} P_{i}^{2}+\frac{\left(\frac{8}{3}\left|\hat{h}_{k k}^{m}\right|^{2} \Delta_{I}^{2}+\frac{7}{90} \Delta_{I}^{4}\right) P_{k}^{2}}{\left(2^{2 R_{k}^{m}}-1\right)^{2}}}} .
$$

Proof: By rearranging (5), we have:

$$
\mathrm{P}_{k}^{\text {out }, m}=\operatorname{Pr}\left\{Y \geq \frac{4\left|\hat{h}_{k k}^{m}\right|^{2} P_{k}}{2^{2 R_{k}^{m}}-1}-2\right\}
$$

in which $\frac{4\left|\hat{h}_{k k}^{m}\right|^{2} P_{k}}{2^{2 R_{k}^{m n}}-1}-2$ is a constant value, and the random variable $Y$ is defined as follows:

$$
\begin{aligned}
Y= & \sum_{l=1, l \neq k}^{M}\left(\Re\left[\delta_{k, l}^{m}+\delta_{k, l}^{m_{p}}\right]^{2}+\Im\left[\delta_{k, l}^{m}+\delta_{k, l}^{m_{p}}\right]^{2}\right) P_{l} \\
- & \frac{4\left(\Re\left[\hat{h}_{k k}^{m}\right] \Re\left[\delta_{k k}^{m}+\delta_{k k}^{m_{p}}\right]+\Im\left[\hat{h}_{k k}^{m}\right] \Im\left[\delta_{k k}^{m}+\delta_{k k}^{m_{p}}\right]\right) P_{k}}{2^{2 R_{k}^{m}}-1} \\
& -\frac{\left(\Re\left[\delta_{k k}^{m}+\delta_{k k}^{m_{p}}\right]^{2}+\Im\left[\delta_{k k}^{m}+\delta_{k k}^{m_{p}}\right]^{2}\right) P_{k}}{2^{2 R_{k}^{m}}-1} .
\end{aligned}
$$

The mean and variance of $Y$ are (calculated in Appendix A):

$$
\begin{aligned}
\mu_{Y} & =\frac{\Delta_{I I}^{2}}{3} \sum_{l=1, l \neq k}^{M} P_{l}-\frac{\Delta_{I}^{2} P_{k}}{3\left(2^{2 R_{k}^{m}}-1\right)}, \\
\sigma_{Y}^{2} & =\frac{7 \Delta_{I I}^{4}}{90} \sum_{l=1, l \neq k}^{M} P_{l}^{2}+\frac{\left(\frac{8}{3}\left|\hat{h}_{k k}^{m}\right|^{2} \Delta_{I}^{2}+\frac{7}{90} \Delta_{I}^{4}\right) P_{k}^{2}}{\left(2^{2 R_{k}^{m}}-1\right)^{2}} .
\end{aligned}
$$

Let $X$ be a random variable with mean $\mu_{X}$ and variance $\sigma_{X}^{2}$. For any real value $r>0$ the Cantelli inequality implies $\operatorname{Pr}\left\{X-\mu_{X} \geq r \sigma_{X}\right\} \leq \frac{1}{1+r^{2}}$ [11]. Setting $X=Y$ and $r_{k}^{m} \sigma_{Y}+\mu_{Y}=\frac{4\left|\hat{h}_{k k}^{m}\right|^{2} P_{k}}{2^{2 R_{k}^{m}}-1}-2$, applying the Cantelli inequality leads to the value of $r_{k}^{m}$ and $R_{\max , k}^{m}$ as in (7) and the upper bound on $\mathrm{P}_{k}^{\text {out }, m}$ as in (6).

This theorem clarifies how the outage probability of each user is dependent on parameters such as the quantized channel gain and transmission rate of that user, the transmission powers, the number of users and the quantization resolutions.

When the quantization resolutions are sufficiently high we have the following Corollary to Theorem 1 .

Corollary 1: If $R_{k}^{m}<\frac{1}{2} \log \left(1+2\left|\hat{h}_{k k}^{m}\right|^{2} P_{k}\right)$, high-resolution quantizers $\left(\Delta_{I}, \Delta_{I I} \rightarrow 0\right)$ lead to $\mathrm{P}_{k}^{\text {out }, m}=0$.

Proof: If $R_{k}^{m}<\frac{1}{2} \log \left(1+2\left|\hat{h}_{k k}^{m}\right|^{2} P_{k}\right), \lim _{\Delta_{I}, \Delta_{I I} \rightarrow 0} r_{k}^{m}=+\infty$ and the upper bound on $\mathrm{P}_{k}^{\text {out }, m}$ approaches 0 . Since, $\mathrm{P}_{k}^{\text {out }, m}$ is lower bounded by 0 we can conclude $\mathrm{P}_{k}^{\text {out }, m} \rightarrow 0$.

This result shows that when the quantizers are fine enough, reliable transmission is possible if the transmission rate $R_{k}^{m}$ is chosen less than $\frac{1}{2} \log \left(1+2\left|\hat{h}_{k k}^{m}\right|^{2} P_{k}\right)$. Therefore, the average rate of $\frac{1}{2} E \log \left(1+2\left|\hat{h}_{k k}^{m}\right|^{2} P_{k}\right)$ can indeed be achieved, which coincides with the result of reference [5].

\section{NETWORK THROUGHPUT}

\section{A. Throughput definition}

We define the throughput as the average rate of successful message delivery. At any pair of complement blocks $m$ and $m_{p}$, the network throughput $T^{m}$ can be represented as the summation over the throughput of the individual users, i.e.

$$
T^{m}=\sum_{k=1}^{M} T_{k}^{m}=\sum_{k=1}^{M} R_{k}^{m}\left(1-\mathrm{P}_{k}^{\text {out }, m}\right),
$$

where $T_{k}^{m}$ is the throughput of the $k^{t h}$ transmitter-receiver pair. Considering the transmission over a large number of blocks, the average throughput of the network is $T=E\left[T^{m}\right]$, in which $E$ is the expectation over channel coefficient matrix. Exploiting the upper bound on $\mathrm{P}_{k}^{\text {out }, m}$ in (6), the throughput can be lower bounded as:

$$
T^{m} \geq \sum_{k=1}^{M} R_{k}^{m}\left(1-\mathrm{P}_{\mathrm{up}, k}^{\mathrm{out}, m}\left(R_{k}^{m}, P_{1}, \ldots, P_{M},\left|\hat{h}_{k k}^{m}\right|\right)\right),
$$

in which $\mathrm{P}_{\mathrm{up}, k}^{\text {out }, m}\left(R_{k}^{m}, P_{1}, \ldots, P_{M},\left|\hat{h}_{k k}^{m}\right|\right)$ is the upper bound on $\mathrm{P}_{k}^{\text {out }, m}$ in the RHS of (6). Since the upper bound $\mathrm{P}_{\mathrm{up}, k}^{\mathrm{out}, m}$ depends only on the desired channel gain, we have the following lower bound on the average network throughput:

$T \geq \sum_{k=1}^{M} E_{k}\left[R_{k}^{m}\left(1-\mathrm{P}_{\text {up }, k}^{\text {out } m}\left(R_{k}^{m}, P_{1}, \ldots, P_{M},\left|\hat{h}_{k k}^{m}\right|\right)\right)\right]$,

in which $E_{k}$ is the expectation over the gain of channel $k$. At each compliment block pair, the lower bound (12) can be maximized by rate adaptation and power allocation. This problem can be formulated as follows:

$$
\max _{\substack{0 \leq P_{i} \leq P_{i}^{\max } \\ 0 \leq R_{i}^{m} \\ i=1, \ldots, M}} \sum_{k=1}^{M} R_{k}^{m}\left(1-\mathrm{P}_{\text {up }, k}^{\text {out }, m}\left(R_{k}^{m}, P_{1}, \ldots, P_{M},\left|\hat{h}_{k k}^{m}\right|\right)\right)
$$


In the following, we study the rate adaptation for a given set of allocated powers.

\section{B. Rate adaptation}

For a given set of transmission powers, since $\mathrm{P}_{\mathrm{up}, k}^{\text {out }, m}$ only depends on $\hat{h}_{k k}^{m}$ and $R_{k}^{m}$ the optimization of the lower bound of the throughput in (14) simplifies as that each transmitter optimizes its throughput individually as follows:

$$
\sum_{k=1}^{M} \max _{0 \leq R_{k}^{m}} R_{k}^{m}\left(1-\mathrm{P}_{\text {up }, k}^{\text {out }, m}\left(R_{k}^{m},\left|\hat{h}_{k k}^{m}\right|\right)\right) .
$$

Substituting the upper bound of $\mathrm{P}_{k}^{\text {out }, m}$ given in (6), each transmitter solves the following optimization problem:

$$
\max _{0 \leq R_{k}^{m} \leq R_{\max , k}^{m}} R_{k}^{m}\left(\frac{\left(r_{k}^{m}\right)^{2}}{1+\left(r_{k}^{m}\right)^{2}}\right)
$$

where, $R_{\max , \mathrm{k}}^{m}$ and $r_{k}^{m}$ are given in (7). After certain mathematical manipulations and introducing a new optimization variable $x$, we have the following equivalent optimization problem:

$$
\max _{\substack{x=2^{2} R_{k}^{m} \\ 0 \leq R_{k}^{m} \leq R_{\max , k}^{m}}} R_{k}^{m} \times \frac{A x^{2}+B x+C}{D x^{2}+E x+F}
$$

where,

$$
\begin{aligned}
& A=\left(-\frac{\Delta_{I I}^{2}}{3} \sum_{i=1, i \neq k}^{M} P_{i}-2\right)^{2}, C=\frac{1}{9}\left(12\left|h_{k k}^{m}\right|^{2}+\Delta_{I}^{2}\right) P_{k}^{2} \\
& B=\frac{2}{3}\left(12\left|h_{k k}^{m}\right|^{2}+\Delta_{I}^{2}\right)\left(-\frac{\Delta_{I I}}{3} \sum_{i=1, i \neq k}^{M} P_{i}-2\right) P_{k} \\
& D=\frac{7}{90} \Delta_{I I}^{4} \sum_{i=1, i \neq k}^{M} P_{i}^{2}+\left(-\frac{\Delta_{I I}^{2}}{3} \sum_{i=1, i \neq k}^{M} P_{i}-2\right)^{2} \\
& F=\frac{1}{9}\left(12\left|h_{k k}^{m}\right|^{2}+\Delta_{I}^{2}\right) P_{k}^{2}+\left(\frac{8}{3}\left|h_{k k}^{m}\right|^{2} \Delta_{I}^{2}+\frac{7}{90} \Delta_{I}^{4}\right) P_{k}^{2} \\
& E=\frac{2}{3}\left(12\left|h_{k k}^{m}\right|^{2}+\Delta_{I}^{2}\right)\left(-\frac{\Delta_{I I}}{3} \sum_{i=1, i \neq k}^{M} P_{i}-2\right) P_{k}
\end{aligned}
$$

This problem is not convex. However, it can be proved that the feasible set of this problem satisfies the linear independent constraint qualification (LICQ) conditions [12] and the duality gap is zero. Therefore, any pair of primal and dual optimal points of this problem must satisfy the KKT conditions. Solving the KKT conditions, the necessary condition on the optimal solution $\left(R_{k}^{m *}, x^{*}\right)$ is [13]:

$$
\begin{aligned}
f\left(x^{*}\right) & =\ln \left(x^{*}+1\right) \\
& +\frac{\left(x^{*}+1\right)^{-1}\left(A x^{* 2}+B x^{*}+C\right)\left(D x^{* 2}+E x^{*}+F\right)}{(A E-D B) x^{* 2}+2(A F-D C) x^{*}+(B F-E C)}=0
\end{aligned}
$$

This equation can be solved by Newton method. Solution for the rate is $R_{k}^{m *}=\frac{1}{2} \log \left(1+x^{*}\right)$.

To obtain a sense on the behavior of the throughput as a function of the transmission rate, as an example we consider a 3-user network. We assume $P_{1}=P_{2}=P_{3}=+\infty$

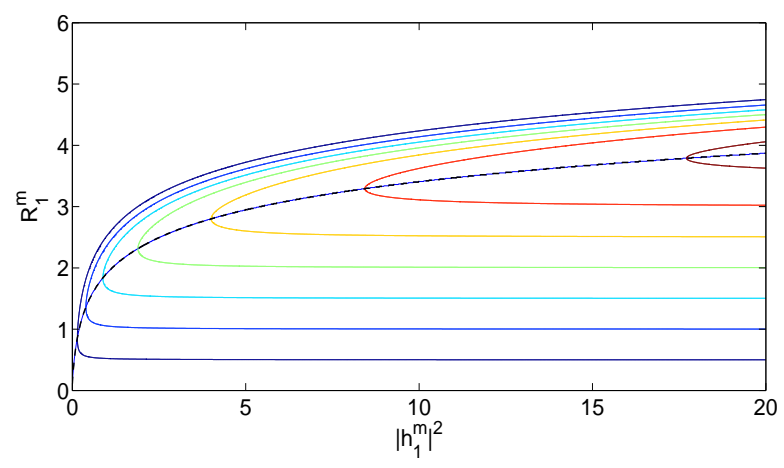

Fig. 2: Throughput of the first user in the 3-user network as a function of rate and channel gain.

and $K_{I}=K_{I I}=3$. The throughput of the first user $R_{1}\left(1-\mathrm{P}_{1}^{\text {out }}\left(R_{1},\left|h_{11}^{m}\right|^{2}\right)\right)$ as a function of the transmission rate for different channel gains is represented in Fig. 2 as contours. The throughput remains the same over each of the curves and increases as the color of the curve goes from the blue to the red tail of the spectrum. This figure shows that for a given quantized channel gain, there is a global maximum. The optimum rate can be derived by solving (19).

\section{Performance eValuation}

In this section, we use numerical results to evaluate the performance of the considered scheme.

As an example, the lower bound on the expected throughput of the 3-user network evaluated assuming $P_{1}=P_{2}=P_{3}=P$. Fig. 3 is based on the assumption that $K_{I}=K_{I I}$. It shows that by increasing the quantization resolution, the throughput approaches that when perfect channel knowledge assumed in reference [5]. At high SNR the throughput saturates, since the power of the residual interference is proportional to the transmission power $P$ and according to (4) as $P$ increases the SINR converges to a limited value.

For the fixed number of the total feedback bits of each receiver $\left(K_{f}\right)$, the lower bound on the throughput in three different scenarios presented in Fig. 4. This shows that in low SNR region more quantization bits should be allocated to the desired channel while at high SNR region allocating more bits to the quantization of the interference channels is preferred. This result intuitively is expected, since in low SNR region noise is the dominant factor and it is better to have more accurate information about the desired channel gain for rate adaptation, while at high SNR region interference is the dominant factor and it is preferred to have more accurate information about the interference channels to perform interference alignment more precisely.

Fig. 5 represents the lower bound on the throughput for different number of users $M$ assuming that $P_{1}=P_{2}=$ $\ldots=P_{M}=P$. Also, performance of the TDMA scheme is shown assuming perfect CSI is available at the transmitters. It shows that the better performance of interference alignment over TDMA may still exist even with partial CSI. 


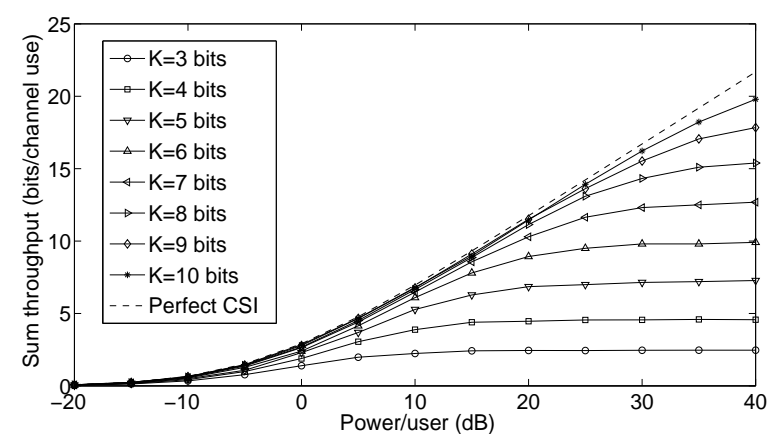

Fig. 3: Throughput of the 3-users network, $K_{I}=K_{I I}=K$.

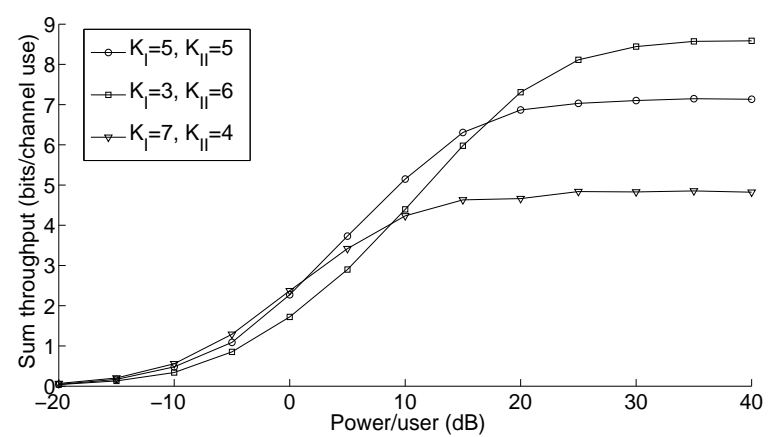

Fig. 4: Throughput of the 3 -user network, $K_{I}+2 K_{I I}=15$.

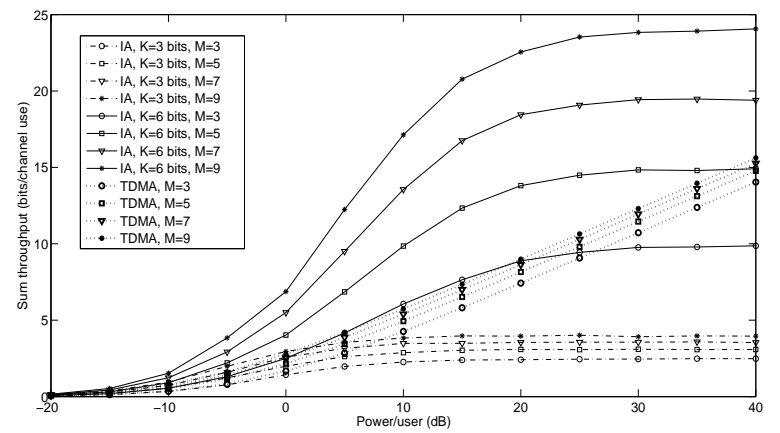

Fig. 5: Throughput of the M-user network.

\section{APPENDIX A \\ PROOF OF THEOREM 1}

In this part, we calculate the mean and variance of random variable $Y$ defined in (9). We exploit the property that quantization error of a Gaussian random variable with variance $\sigma^{2}$ is uniformly distributed with an acceptable approximation when $\sigma / \Delta \geq 1$ in which $\Delta$ is the quantizer step size [14]. Therefore, we assume uniform distribution for variables: $\Re\left[\delta_{k l}\right], \Im\left[\delta_{k l}\right], \Re\left[\delta_{k l}^{p}\right], \Im\left[\delta_{k l}^{p}\right] \sim U\left(-\frac{\Delta}{2}, \frac{\Delta}{2}\right)$, in which $\Delta=\frac{h_{\max }}{2^{K-1}}$ for $K$ bit quantization when the magnitude of the real and imaginary parts of channel gains are limited to $h_{\max }$.

We define random variables $g_{k l}^{\Re}$ and $g_{k l}^{\Im}$ as follow:

$$
g_{k l}^{\Re}=\Re\left[\delta_{k l}+\delta_{k l}^{p}\right], g_{k l}^{\Im}=\Im\left[\delta_{k l}+\delta_{k l}^{p}\right]
$$

Assuming uniform distribution for quantization errors, since $f_{X+Y}=f_{X} * f_{Y}$ in which ${ }^{*}$ ' is the convolution operation, the probability density functions (pdfs) of these variables are:

$$
f_{g_{k l}^{\Re}}(x)=f_{g_{k l}^{\Im}}(x)=\frac{1}{\Delta^{2}}(\Delta-|x|) ; 0<|x|<\Delta
$$

Mean and variance of these random variables are as follows:

$$
E\left[g_{k l}^{\Re}\right]=E\left[g_{k l}^{\Im}\right]=0, \operatorname{var}\left[g_{k l}^{\Re}\right]=\operatorname{var}\left[g_{k l}^{\Im}\right]=\frac{\Delta^{2}}{6}
$$

Also, we define random variables $s_{k l}^{\Re}$ and $s_{k l}^{\Im}$ as follow:

$$
s_{k l}^{\Re}=\left(\Re\left[\delta_{k l}+\delta_{k l}^{p}\right]\right)^{2}, s_{k l}^{\Im}=\left(\Im\left[\delta_{k l}+\delta_{k l}^{p}\right]\right)^{2}
$$

If $Y=g(X), f_{Y}(y)=\sum_{k=1}^{n(y)} 1 /\left|g^{\prime}\left(g_{k}^{-1}(y)\right)\right| \cdot f_{X}\left(g_{k}^{-1}(y)\right)$ where $n(y)$ is the number of solutions in $x$ for the equation $g(x)=y$, and $g_{k}^{-1}(y)$ are these solutions, exploiting (21) pdfs of these random variables are as follows:

$$
f_{s_{k l}^{\Re}}(x)=f_{s_{k l}^{\Im}}(x)=\frac{1}{\Delta \sqrt{x}}-\frac{1}{\Delta^{2}} ; \quad 0<x<\Delta^{2}
$$

Mean and variance of these random variables are as follow:

$$
E\left[s_{k l}^{\Re}\right]=E\left[s_{k l}^{\Im}\right]=\frac{\Delta^{2}}{6}, \operatorname{var}\left[s_{k l}^{\Re}\right]=\operatorname{var}\left[s_{k l}^{\Im}\right]=\frac{7 \Delta^{4}}{180}
$$

According to (9), (25) and (22), we have the mean and variance values given in (10).

\section{ACKNOWLEDGMENT}

The authors wish to thank Majid Nasiri Khormuji for helpful discussions on this work.

\section{REFERENCES}

[1] G. Kramer, "Outer bounds on the capacity of Gaussian interference channels," IEEE Trans. Inform. Theory, vol. 50, no. 3, pp. $581-586$, 2004.

[2] T. Han and K. Kobayashi, "A new achievable rate region for the interference channel," Communication Over MIMO X Channels: Interference Alignment, Decomposition, and Performance Analysis, vol. 27, no. 1 pp. 49-60, 1981.

[3] V. R. Cadambe and S. A. Jafar, "Interference alignment and degrees of freedom of the K-user interference channel," IEEE Trans. Inform. Theory, vol. 54, no. 8, pp. $3425-3441,2008$.

[4] M. A. Maddah-Ali, A. S. Motahari, and A. K. Khandani, "Communication over mimo x channels: Interference alignment, decomposition, and performance analysis," IEEE Trans. Inform. Theory, vol. 54, pp. 3457 - 3470, 2008.

[5] B. Nazer, S. Jafar, M. Gaspar, and S. Vishwanath, "Ergodic interference alignment," in IEEE Int. Symp. Information Theory (ISIT'09), Seoul, Korea, 2009.

[6] H. Bolcskei and I. J. Thukral, "Interference alignment with limited feedback," in IEEE Int. Symp. Information Theory (ISIT'09), Seoul, Korea, 2009, pp. $1759-1763$.

[7] R. T. Krishnamachari and M. K. Varanasi, "Interference alignment under limited feedback for MIMO interference channels," in IEEE Int. Symp. Information Theory (ISIT'10), 2010, pp. 619 -623.

[8] R. M. Gray and D. L. Neuhoff, "Quantization," IEEE Trans. Inform. Theory, vol. 44, no. 6, pp. 2325 -2383, Oct. 1998.

[9] J. Max, "Quantizing for minimum distortion," IEEE Trans. Inform. Theory, vol. 6, no. 1, pp. $7-12,1960$.

[10] D. Tse and P. Viswanath, Fundamentals of wireless communication. Cambridge Univ. Press, 2005.

[11] C. L. Mallows and D. Richter, "Inequalities of Chebyshev type involving conditional expectations," The Annals of Mathematical Statistics, vol. 40 , no. 6, pp. 1922-1932, 1969.

[12] R. Heniron, "On constraint qualifications," J. Optim. Theory Appl., vol. 72 , no. $1,1992$.

[13] S. Boyd and L. Vanderbenghe, Convex optimization. Cambridge Univ. Press, 2004

[14] A. Sripad and D. Snyder, "A necessary and sufficient condition for quantization errors to be uniform and white," IEEE Transactions on Acoustics, Speech and Signal Processing, vol. 25, no. 5, pp. 442 - 448, Oct. 1977. 